\title{
Convolutional Neural Network for Seizure Detection of Nocturnal Frontal Lobe Epilepsy
}

\author{
Fabio Pisano $\mathbb{D}^{1},{ }^{1}$ Giuliana Sias $\left(\mathbb{D},{ }^{1}\right.$ Alessandra Fanni $\left(\mathbb{D},{ }^{1}\right.$ Barbara Cannas, ${ }^{1}$ \\ António Dourado $\mathbb{D}^{2}{ }^{2}$ Barbara Pisano, ${ }^{1}$ and Cesar A. Teixeira $\mathbb{D i D}^{2}$ \\ ${ }^{1}$ Department of Electrical and Electronic Engineering, University of Cagliari, Cagliari 09123, Italy \\ ${ }^{2}$ Univ Coimbra, CISUC-Center for Informatics and Systems of the University of Coimbra, \\ Department of Informatics Engineering, Coimbra, Portugal
}

Correspondence should be addressed to Fabio Pisano; fabio.pisano@diee.unica.it

Received 9 September 2019; Accepted 22 February 2020; Published 31 March 2020

Guest Editor: Murari Andrea

Copyright (C) 2020 Fabio Pisano et al. This is an open access article distributed under the Creative Commons Attribution License, which permits unrestricted use, distribution, and reproduction in any medium, provided the original work is properly cited.

\begin{abstract}
The Nocturnal Frontal Lobe Epilepsy (NFLE) is a form of epilepsy in which seizures occur predominantly during sleep. In other forms of epilepsy, the commonly used clinical approach mainly involves manual inspection of encephalography (EEG) signals, a laborious and time-consuming process which often requires the contribution of more than one experienced neurologist. In the last decades, numerous approaches to automate this detection have been proposed and, more recently, machine learning has shown very promising performance. In this paper, an original Convolutional Neural Network (CNN) architecture is proposed to develop patient-specific seizure detection models for three patients affected by NFLE. The performances, in terms of accuracy, sensitivity, and specificity, exceed by several percentage points those in the most recent literature. The capability of the patient-specific models has been also tested to compare the obtained seizure onset times with those provided by the neurologists, with encouraging results. Moreover, the same CNN architecture has been used to develop a cross-patient seizure detection system, resorting to the transfer-learning paradigm. Starting from a patient-specific model, few data from a new patient are enough to customize his model. This contribution aims to alleviate the task of neurologists, who may have a robust indication to corroborate their clinical conclusions.
\end{abstract}

\section{Introduction}

Nocturnal frontal lobe epilepsy (NFLE) is a rare form of epilepsy, typically inherited, which affects both sexes from 1 to 60 years, and is associated with cognitive decline. Crises are characterized by frequent and brief hypermotor sleep seizures, which may range from a simple awakening from sleep to more focused motor movements, with dystonic and tonic postures, grimaces, screams or groans, episodic nocturnal wanderings, and stereotyped agitated somnambulism, among others $[1,2]$. In this paper, the problem of detecting NFLE seizures, using Electroencephalographic (EEG) signals as inputs to an original Convolutional Neural Network (CNN) prediction model, is addressed.

The use of EEG signals is corroborated by most of the literature on epileptic seizure detection, which considers indeed the EEG the gold standard to analyse the electrical activity of the brain [3]

In the literature, there is a wide range of proposals for the identification of general epileptic seizures [4-13], mostly based on the machine-learning approach, which support the doctors in the time-consuming manual labelling [4]. However, very few contributions specifically dealing with NFLE are present, and they reach lower performance indices. In [14], a detection system for NFLE seizures has been proposed, which is based on accelerometer signals, obtaining a value of sensitivity of $91.67 \%$ and specificity of $84.19 \%$ on three pediatric patients. The accelerometers were already proposed in $[15,16]$ to detect epileptic seizures with pros and cons; even if they are more comfortable to wear, they can only reveal seizures associated to motor activity. Moreover, high false-positive rates are often possible due to motion 
artifacts that cannot be readily distinguished from convulsions. In [17], a machine-learning technique has been proposed to classify NFLE seizures and nonseizures using the C4/A1 EEG channel (according to the International 10-20 System [18]), which is one of the monopolar derivations currently used for the conventional sleep staging and arousal scoring [19], obtaining a value of specificity of $82.4 \%$. In [20], the Self-Organizing Maps (SOMs) are used to develop patient-specific seizure detection systems for NFLE. The proposed method allows the construction of maps that contain important information on the current brain state, showing the potential of the map to correctly cluster data from seizure and nonseizure patterns. In addition, it also suggests the use of SOMs for early crisis detection by projecting the EEG trace onto it. Following the trajectory of this projection on the map, it is possible to activate an alarm depending on the composition of the cluster where the current EEG sample is projected. The method has been tested on nine patients obtaining a mean value of sensitivity equal to $80.5 \%$ and a mean value of specificity equal to $90.2 \%$.

Despite these quite good results, the cited literature shows that the feature engineering phase is crucial for the success of the machine-learning predictive models and the identification of proper seizure indicators is a preliminary due diligence. In fact, it highly influences the performance of the classifier and it is often conditioned to a specific knowledge and competencies on the pathophysiology under study. Moreover, extracting features is usually carried out by hand and it is very time consuming, requiring a high number of scenarios simulations in order to choose the best combination of features. To avoid this challenging phase, in the present paper, CNNs have been used. In fact, CNNs are able to automatically learn relevant low-dimensional features from high-dimensional data by successively applying convolutional and downsampling operations.

$\mathrm{CNNs}$, which belong to the so-called deep neural networks [21-23], are now affirming themselves for the automated detection of epileptic seizures from EEG signals. In [24], Acharya et al. proposed a one-dimensional (1-D) CNN with 13 layers: 5 convolutional layers, 5 max-pooling layers, and 3 fully connected layers. The CNN has been trained to classify EEG epochs in three classes: normal, preictal, and seizure. Using 100 single channel EEG traces of five patients from the Bonn EEG database [25], an accuracy of $88.67 \%$, a sensitivity of $95 \%$, and a specificity of $90 \%$ are obtained with a ten-fold crossvalidation. Even if the results are promising, comparison with the literature on the same database revealed that the proposed detection system could be improved provided that more EEG data are available. In [26], Ullah et al. proposed an ensemble of pyramidal 1-D CNNs (P-1D-CNN) to solve the same three classes' detection problem addressed in [24] on the same University of Bonn database. Each P-1D-CNN has three convolutional layers and two fully connected layers. Several simulations have been performed to select the best model parameters. In order to overcome the lack of data, already highlighted in [24], two data augmentation schemes have been introduced. A majority voting strategy has been adopted to fuse the decisions of the ensemble of CNNs, reaching an average accuracy of $96.1 \%$. In [27], the authors exploited the attitude of the $\mathrm{CNNs}$ to process images, rather than $0-\mathrm{D}$ temporal sequences. For this purpose, the raw EEG multichannels data have been firstly filtered, then segmented using a prefixed time window, and finally converted into a series of EEG-plot images. The proposed CNN, which is based on the VGG-16 architecture of [28], has been trained to classify each image into seizure or nonseizure class. The results, presented in terms of median true positive rate labelling by seconds is $74 \%$, which the authors claimed to be higher than that of commercially available seizure detection software. In [29], each single-channel EEG signal is converted into a $2 \mathrm{D}$ plot and the plots corresponding to 22 different EEG channels are combined in $3 \mathrm{D}$ images depending on the mutual correlation of the intensities between the electrodes. Then, using 3D kernels, a 3D CNN, with 4 convolutional layers, 3 max pooling layers, and 2 fully connected layers, has been built to detect interictal, preictal, and ictal stages in 13 epileptic patients. An accuracy of $90 \%$, a sensitivity of $88.9 \%$, and a specificity of $93.8 \%$ is obtained using a ten-fold crossvalidation procedure.

As shown in the cited references, training CNN models requires a huge amount of EEG records manually labelled by skilled neurologists, which may be a tedious and timeconsuming task. Hence, as highlighted by most of the authors, the major issues limiting the performance of these deep-learning seizure detection methods are the amount of training data, which may not be enough, and the feature space of seizures, which may vary significantly across different patient EEG recordings and even for the same patient. To overcome these limitations, in the present paper, a transfer-learning strategy, which can significantly reduce the quantity of data needed for training, is adopted. A survey on the transfer learning is reported in [30], where it is defined as "the ability of a system to recognize and apply knowledge and skills learned in previous tasks to novel tasks." Transfer learning proved to be particularly useful in training deep neural networks that can share some lower layers of a network previously trained with data from other tasks, while the upper layers are retrained and fine-tuned using taskdependent data [31]. Transfer-learning techniques are still largely unexplored although they seem to have a great potential to overcome the issue of lack of data available for epileptic seizure detection.

In the present paper, the CNNs have been firstly used to build patient-specific seizure detection systems for three patients affected by NFLE. The detection problem has been modelled as a seizure and nonseizure classification problem, obtaining performances that overcome those of other methods presented in [20] for the same patients. The patientspecific CNN model has been used also to find the seizure onset, limiting the time-consuming manual labelling for doctors. Then, a transfer-learning technique has been adopted, allowing to fine tune one of the CNN models, trained with data from one patient, to obtain a detection predictor for a different patient. The results obtained in the present work indicate that even just a seizure is enough to customize the CNN to the new patient. 
The rest of this paper is organized as follows. The database used, the data augmentation schema, and CNN model architecture are discussed in Section 2. Section 3 presents and discusses the results obtained by the patient-specific and the cross-patient detection systems. Section 4 concludes the findings of the study.

\section{Materials and Methods}

A combination of deep learning and transfer learning has been used to build patient-specific and cross-patient seizure detection systems. The detection problem has been formalized as a two-class classification problem, where the two classes correspond to seizure (SZ) or nonseizure (NS) states during the sleep and has been approached by training Convolutional Neural Network models with long-term EEG recordings including epileptic seizures.

2.1. Dataset. Data for this study comes from two epilepsy centers: Unidade de Monitorização em Epilepsia e Sono do Centro Hospitalar e Universitário de Coimbra, Portugal, Epilepsiezentrum, Universitätsklinikum Freiburg, Germany, on behalf of the EPILEPSIAE project [32]. The data concern three patients affected by nocturnal seizures located in the frontal lobe (NFLE). The same patients have been considered also in [20], and the subject numbers refer to those in Table 1 of [20]. The relevant information on the patients enrolled in this study is summarized in Table 1.

The initial and final time of a seizure (SZ) have been identified based on the agreement between at least two expert neurologists. For the nonseizure recordings, no information is available about the patient sleeping or awake state. As in [20], also in the present study, it has been hypothesized that the patients were sleeping in the ten minutes preceding a seizure and these ten minutes are considered to characterize a nonseizure state (NS).

The dataset includes eleven scalp EEG channels, placed according to the International 10-20 System [33] with a sampling rate of $256 \mathrm{~Hz}$. According to the neurologists' suggestions, the eleven selected channels, coloured in blue in Figure 1, are those more suitable for the NFLE seizure detection: three central channels $(\mathrm{C} 3, \mathrm{C} 4$, and $\mathrm{Cz})$, two frontal channels (F3 and F4), two frontal-polar channels (Fp1 and Fp2), two parietal channels (P3 and P4), and two occipital channels (O1 and O2). All the channels are unipolar derivations with a common reference electrode.

2.2. EEG Segmentation and Data Augmentation. Data, coming from the continuous 11 recordings, each one corresponding to an EEG channel, have been segmented using an overlapping sliding window of 5 seconds. In this way, each time window, or segment, has 1280 data points in the $11 \mathrm{D}$ space. The data segmentation allows to increase the number of training samples, which is mandatory, especially in deep learning $[26,29]$. For the training of the network, the data from seizures were segmented with an overlap with the previous window of $0.1484 \mathrm{~s}, 0.5508 \mathrm{~s}, 1 \mathrm{~s}$, for patient \#5, patient \#6, and patient \#9, respectively. The overlap times during seizures for the three patients have been differently chosen in order to reduce the class unbalance due to different total seizure times (see Table 1). As the mean seizure time is about $10 \%$ of the nonseizure recordings, the length of the nonseizure segments is still equal to 5 seconds without overlapping, whereas, for the seizure segments, the overlap time has been dynamically set to produce a balanced number of segments within the two classes. This operation can produce windows with very similar data, so with few novelties for the CNN training; however, in our opinion, it seems to be the most effective way to balance the classes while maintaining a high quantity of data for deep learning. During testing, a sliding window of $5 \mathrm{~s}$ with an overlap of 1 second, for all patients and both phases (seizure/nonseizure), has been used.

In order to improve the quality of the raw EEG signals, a preprocessing step has been performed. After discarding the windows with missing data, a standard thresholding of potential values has been performed. Here, data have been labelled as artifacts if the absolute value of any data point in the trial exceeded a fixed threshold. This method is currently the most widely used artifact detection method in the EEG community. It is most effective for detecting gross eye blinks or eye movement artifacts. Since even a small number of these extreme values can lead to inaccurate results, the segments including these extreme values have been discarded. Furthermore, filtering has been a necessary step due to the presence of $50 \mathrm{~Hz}$ line noise and DC offset (removal of the $0 \mathrm{~Hz}$ ). Thus, a Notch filter at $50 \mathrm{~Hz}$ with bandwidth $1 \mathrm{~Hz}$ has been applied to remove $50 \mathrm{~Hz}$ line noise, and the average value of each electrode along the preseizure phase has been removed as DC offset. The cleaned dataset for each patient has been then partitioned, in order to randomly assign the different seizures to the training, validation, and test sets.

2.3. CNN Architecture. For the development of the CNN architecture, the deep residual network (ResNet) structure [34] has been adapted for the problem of seizure detection. The CNN architecture is shown in Figure 2.

A first convolutional unit $\left(\mathrm{CU}_{0}\right)$, made up of three layers, transforms the input image of size $1280 \times 11$ into a 4 channels image of size $320 \times 11$. The responsible of the size reduction is the convolutional layer, having filters of size $5 \times 1$ and stride $4 \times 1$. A max pooling layer (MP), with pool size $5 \times 1$ and stride $4 \times 1$, follows the first convolutional unit, reducing again the image dimension to a 4-channel image of size $80 \times 11$. A second convolutional unit $\left(\mathrm{CU}_{1}\right)$ made up of seven layers filters the 4 -channel image of size $80 \times 11$ by means of a residual connection. Two following convolutional units $\left(\mathrm{CU}_{2}\right.$ and $\left.\mathrm{CU}_{3}\right)$, each one made up of nine layers, filter out the 4 -channel image of size $80 \times 11$ by means of a residual connection, reducing the image dimension first to a 16-channel image of size $20 \times 11$ and then to a 64-channel image of size $5 \times 11$. An average pooling layer (AP), with pool size $5 \times 1$, reduces the image dimension to a 64-channel image of size $1 \times 11$, producing a set of 704 features. Finally, a fully connected layer (FC) processes the 704 features, output 
TABle 1: Patients from European epilepsy database.

\begin{tabular}{lcccccc}
\hline Patient & Sex & \# of SZ & SZ time (mean) $(\mathrm{s})$ & Total SZ time (s) & Total record time (s) & Epilepsy center \\
\hline Pat \#5 & M & 5 & 38.00 & 190.00 & 3190.00 & Coimbra \\
Pat \#6 & F & 8 & 89.66 & 717.29 & 5517.29 & Freiburg \\
Pat \#9 & M & 15 & 84.38 & 1265.75 & 10265.75 & Freiburg \\
\hline
\end{tabular}

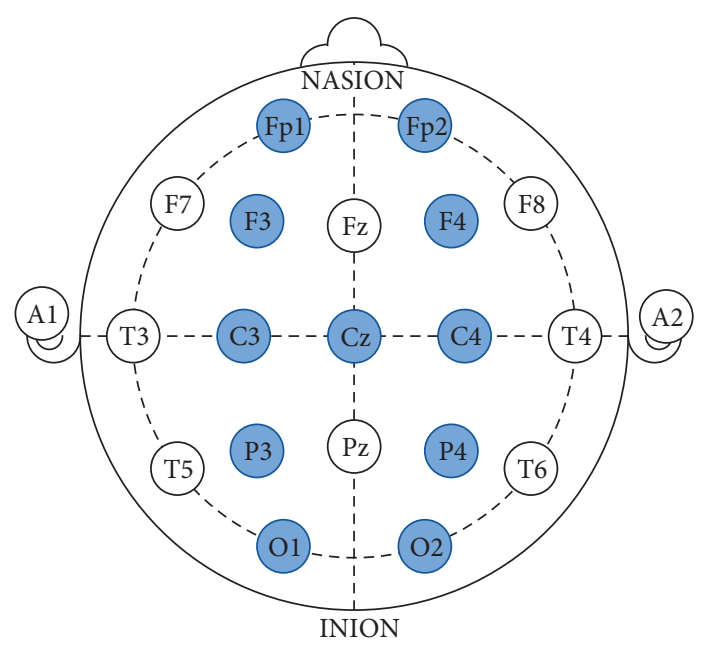

Figure 1: The International 10-20 System [33] with the 11 selected channels (in blue).
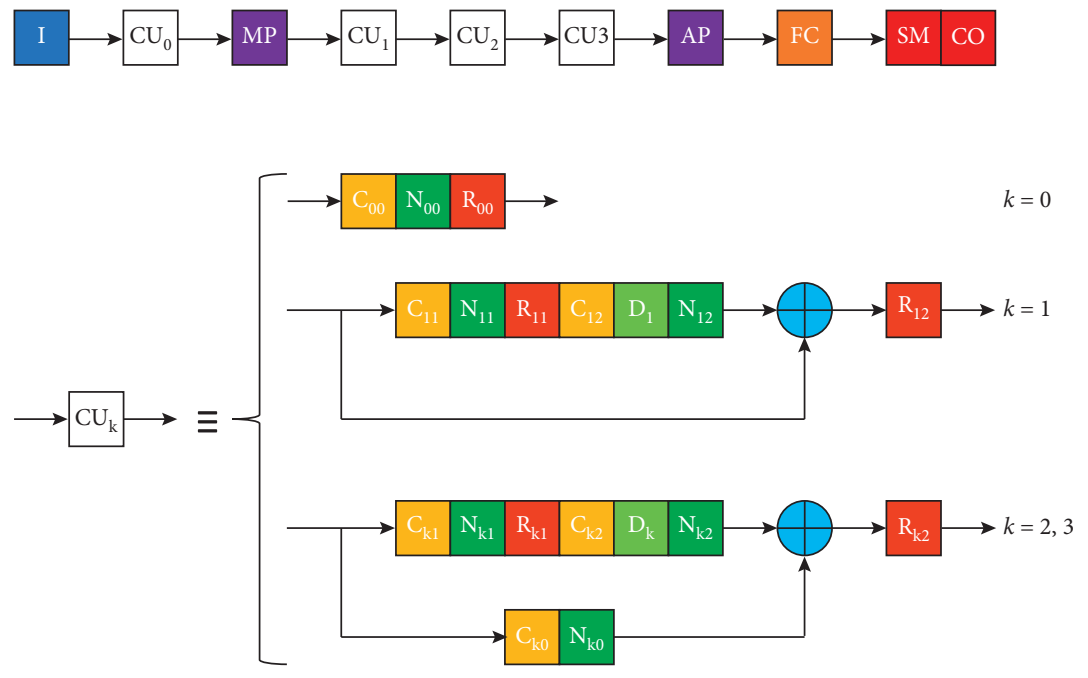

FIGURE 2: CNN architecture, where I is the image input layer; $\mathrm{CU}_{\mathrm{k}}$ is the $k^{\text {th }}$ convolutional unit; $\mathrm{MP}$ and $\mathrm{AP}$ are the max pooling and average pooling layers, respectively; FC is the fully connected layer; and SM and CO are the SoftMax and classification output layer, respectively. In the $k^{\text {th }}$ convolutional unit, $\mathrm{C}_{\mathrm{kj}}, \mathrm{N}_{\mathrm{kj}}, \mathrm{R}_{\mathrm{kj}}$, and $\mathrm{D}_{\mathrm{k}}$ are the $j^{\text {th }}$ convolutional layer, the $j^{\text {th }}$ batch normalization layer, the $j^{\text {th }} \mathrm{ReLu}$ layer, and the dropout layer, respectively.

of the average pooling layer, and feeds a SoftMax layer (SM) for classification (CO).

In this architecture, each channel of the input image is filtered separately, obtaining a set of 64 features per electrode. Features coming from different electrodes are combined in the fully connected layer, i.e., only after the convolutional units, for classification.

Dropout layers with dropout probability of $20 \%$ have been included inside $\mathrm{CU}_{1}, \mathrm{CU}_{2}$, and $\mathrm{CU}_{3}$ convolutional units in order to reduce overfitting on the training set and improve generalization. The algorithm used to train the network is the stochastic gradient descent with momentum [35].

\section{Results and Discussion}

The performance of the deep models has been evaluated using some metrics employed in most of the literature on epilepsy and, in general, in classification by machine learning, such as Accuracy, Specificity, Sensitivity, and Gmean: 


$$
\begin{aligned}
\operatorname{Accuracy}(\mathrm{AC}) & =\frac{\mathrm{TP}+\mathrm{TN}}{\mathrm{TP}+\mathrm{TN}+\mathrm{FP}+\mathrm{FN}}, \\
\operatorname{Specificity}(\mathrm{SP}) & =\frac{\mathrm{TN}}{\mathrm{TN}+\mathrm{FP}}, \\
\text { Sensitivity }(\mathrm{SS}) & =\frac{\mathrm{TP}}{\mathrm{TP}+\mathrm{FN}}, \\
\text { Gmean } & =\sqrt{\text { Specificity } \times \text { Sensitivity, }}
\end{aligned}
$$

where TP (True Positives) are the seizure patterns (SZ) correctly classified, TN (True Negatives) are the nonseizure patterns (NS) correctly classified, FP (False Positives) are the nonseizure patterns classified as seizure patterns, and FN (False Negatives) are the seizure patterns classified as nonseizure patterns.

3.1. Patient-Specific Seizure Detection. Firstly, patient-specific seizure detection systems have been developed for each of the three patients. Table 2 reports the number of seizures and the number of segments used for training, validating, and testing. MATLAB R2018b deep-learning library has been used to develop the CNN models. To train the models an Intel ${ }^{\circledR}$ Core $^{\mathrm{TM}}$ i7 CPU with 16 GB memory has been used, with the help of a Nvidia GeForce GTX 960M with 4 GB memory.

As an example, Figure 3 shows the performance for patient $\# 9$, in terms of accuracy on training, validation, and test sets, against training epochs. The training procedure continues epoch after epoch only if the validation performance increases with respect to the previous epoch. If not, the training is interrupted. This very strong constraint helps avoiding overfitting on the training set, which would occur if the usual crossvalidation procedure is adopted, due to the big size of the network. As shown in Figure 3, for patient \#9, the training has been stopped at the $8^{\text {th }}$ epoch since no better performance has been obtained on the validation set. The training time of the patient-specific models range from approximately 1 hour to 5 hours depending on the seizures in the training and validation sets.

Table 3 reports the performance in terms of Accuracy, Specificity, and Sensitivity for the three patients.

Values greater than $94 \%$ have been achieved for all the performance indices of all the patients with mean values higher than $93 \%$, except for sensitivity related to patient \#9 which is equal to $83.94 \%$. From Table 3, it can be seen that patient $\# 6$ presents very high values for all the indices, reaching even $100 \%$ of specificity. Patient $\# 5$ still has quite high-performance values, even if it is less than the previous case, due to relatively low value of specificity, whereas patient \#9 presents an opposite behaviour with a high value of specificity and the lowest value of sensitivity, showing that EEG features vary from one patient to the other.

For each patient, the obtained results, in terms of geometric mean of specificity and sensitivity (Gmean), have been compared with those in [20], where SOM and SVM have been used as classifiers. This last method is widely used
TABLE 2: Dataset composition for training, validation, and test.

\begin{tabular}{lccccccc}
\hline & $\begin{array}{c}\# \\
\text { of }\end{array}$ & $\begin{array}{c}\text { S of } \\
\text { SZ }\end{array}$ & $\begin{array}{c}\text { A of } \\
\text { SZ }\end{array}$ & $\begin{array}{c}\text { SZ of } \\
\text { SZ }\end{array}$ & $\begin{array}{c}\text { \# of } \\
\text { segment } \\
\text { train }\end{array}$ & $\begin{array}{c}\text { \# of } \\
\text { segment } \\
\text { valid }\end{array}$ & $\begin{array}{c}\text { test of } \\
\text { segment } \\
\text { test }\end{array}$ \\
\hline Pat \#5 & 5 & 3 & 1 & 1 & 1205 & 421 & 645 \\
Pat \#6 & 8 & 4 & 2 & 2 & 1193 & 710 & 1319 \\
Pat \#9 & 15 & 7 & 3 & 5 & 1009 & 486 & 3313 \\
\hline
\end{tabular}

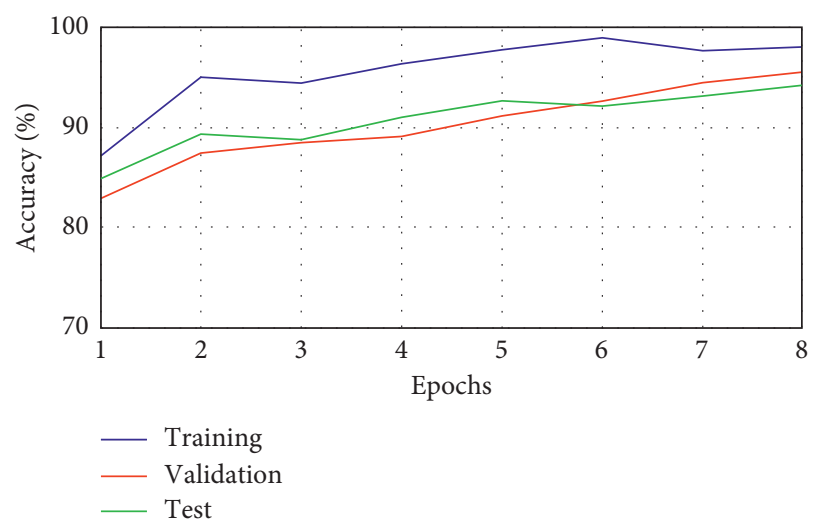

FIgUre 3: Accuracy on training, validation, and test set for patient \#9 against training epochs.

TABle 3: Test performance (\%) of the patient-specific models.

\begin{tabular}{lcccc}
\hline Patient & Pat \#5 & Pat \#6 & Pat \#9 & Mean values \\
\hline Accuracy & 94.42 & 99.77 & 94.99 & 96.39 \\
Specificity & 94.12 & 100 & 96.32 & 96.81 \\
Sensitivity & 98.00 & 97.67 & 83.94 & 93.20 \\
\hline
\end{tabular}

TABle 4: Comparison of Gmean (\%) with some state-of-the-art techniques.

\begin{tabular}{llll}
\hline Method & SOM & SVM & CNN \\
\hline Pat \#5 & 86.02 & 87.05 & 96.04 \\
Pat \#6 & 90.57 & 89.84 & 98.83 \\
Pat \#9 & 82.92 & 82.13 & 89.92 \\
\hline
\end{tabular}

in epileptic seizure detection systems $[4,36]$. The results reported in Table 4 show that CNN improves the performance of both the methods by several percentage points.

These patient-specific models can be used to support the neurologists in the time-consuming identification of the seizure onset time. In particular, during the evolution of the EEG recordings, the condition derived from the CNN model is used to trigger the alarm (i.e., the seizure onset) when the sliding window is classified as SZ for at least $d$ consecutive seconds. The assertion time $d$ has been optimized on the same set used to train the model. As an example, Table 5 reports the difference $\Delta t$ in seconds between the alarm time given by the algorithm $\left(t_{\text {alarm }}\right)$ and the seizure onset time provided by the neurologists $\left(t_{\mathrm{SZ}}\right)$ for the EEG recordings of the patient \# 9 . As it can be noted, in $87 \%$ of cases, the alarm time differs from the seizure onset time by less than $10 \mathrm{~s}$. In the other two cases, this difference is at most $17 \mathrm{~s}$. Figure 4 reports the cumulative alarm time 
TABle 5: Difference $\Delta t(s)$ between the alarm time given by CNN and the seizure onset time provided by the neurologists for the three patients.

\begin{tabular}{|c|c|c|c|c|c|c|c|c|c|c|c|c|c|c|c|}
\hline $\mathrm{SZ} \#$ & 1 & 2 & 3 & 4 & 5 & 6 & 7 & 8 & 9 & 10 & 11 & 12 & 13 & 14 & 15 \\
\hline$\Delta t(s)$ & -4 & -2 & 17 & -3 & -7 & 14 & 1 & -3 & 10 & 0 & 5 & -3 & -1 & -5 & 0 \\
\hline
\end{tabular}

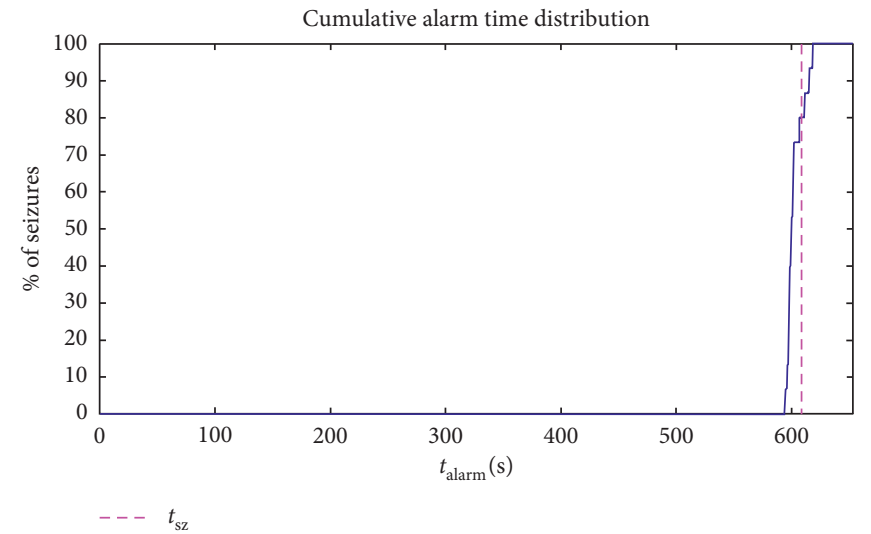

FIgUre 4: Cumulative alarm time distribution for patient \#9. The dashed vertical line refers to the seizure onset time provided by the neurologists.
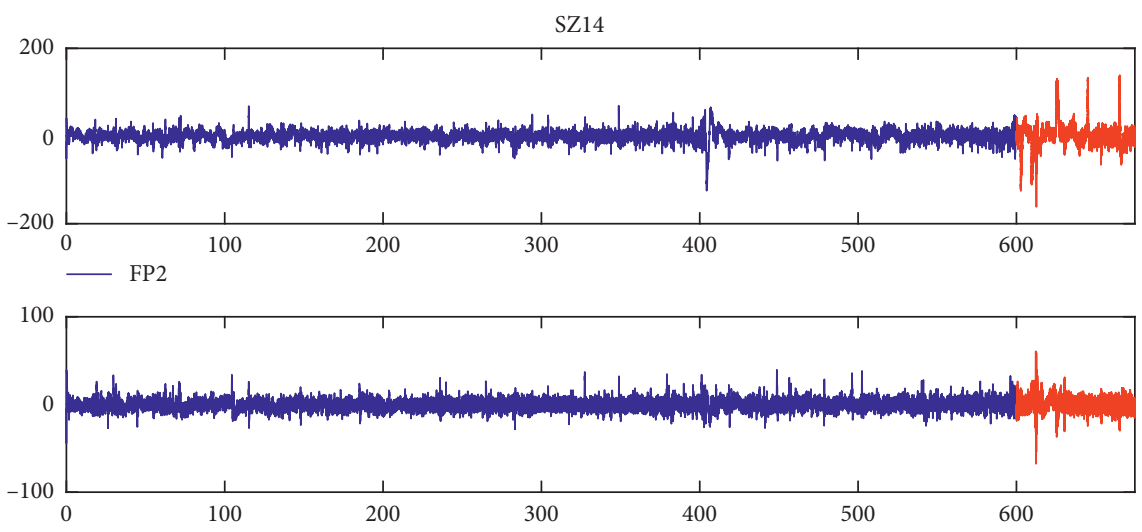

F4

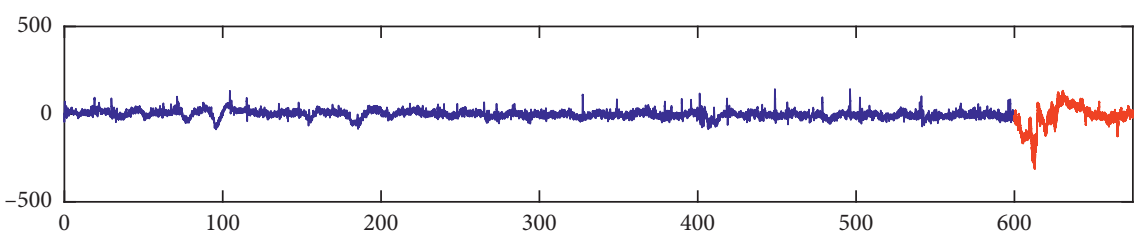

C4



FIGURE 5: Example of the test seizure \#14 of patient \#9. Top three plots: raw signals of the channels FP2, F4, and C4 coloured on the basis of the NS and SZ state; bottom plot: bar graph of the answer of the CNN model, where a blue bar represents a pattern classified as NS, whereas a red bar characterizes a SZ pattern. The green vertical dashed line identifies the onset seizure time given by the CNN $\left(t_{\text {alarm }}\right)$. 
TABle 6: Test Performance (\%) of the cross-patient seizure detection system of patient \#5.

\begin{tabular}{lccccc}
\hline & SZ \#1 & SZ \#2 & SZ \#3 & SZ \#4 & SZ \#5 \\
\hline Accuracy & 41.57 & 93.47 & 90.97 & 91.44 & 98.32 \\
Specificity & 28.03 & 85.92 & 95.70 & 85.56 & 98.22 \\
Sensitivity & 100 & 100 & 81.69 & 97.47 & 91.32 \\
Gmean & 52.94 & 92.69 & 88.42 & 99.11 \\
\hline
\end{tabular}

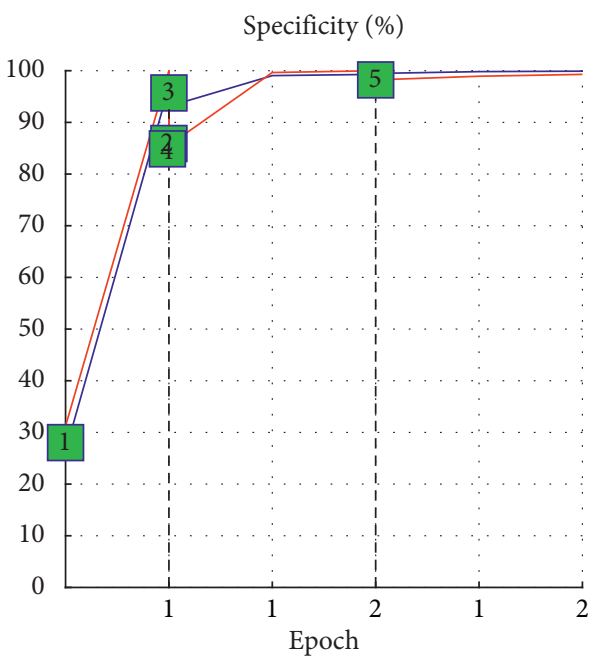

(a)

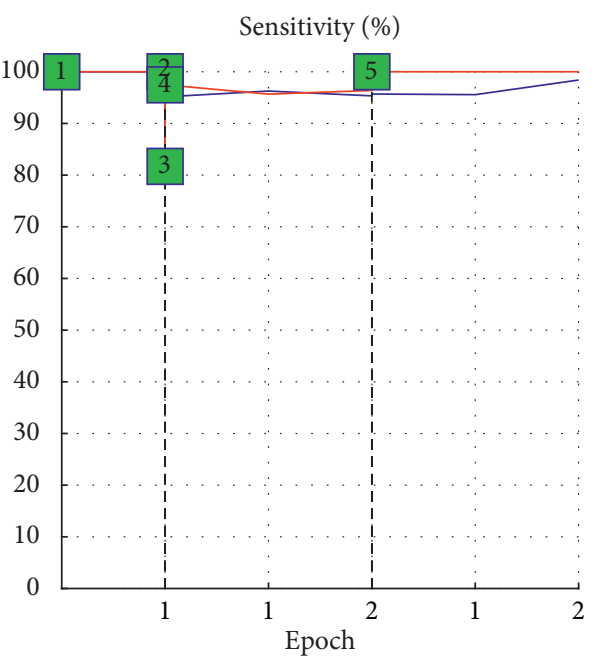

(b)

Figure 6: Results of the cross-patient seizure detection procedure in terms of Specificity (a) and Sensitivity (b), starting from the seizure detection model of patient \#9 and fine-tuning it for patient \#5. Figure shows the trend of Specificity and Sensitivity during the temporal occurrence of the 5 seizures of patient \#5. Blue line: trend on the training set; red line: trend on the validation set; green squares: results on the test set.

distribution for patient \#9. In the same figure, the seizure onset time provided by the neurologists is reported. Note that, because in each EEG recordings the first 10 minutes correspond to nonseizure states (NS), $t_{\mathrm{SZ}}$ is always equal to $600 \mathrm{~s}$.

Figure 5 shows, as an example, the testing seizure \#14 of patient \#9 and the preceding $10 \mathrm{~min}$. The three top plots in Figure 5 report the raw signals of the channels FP2, F4, and C4, which have been colored in blue for the NS state and in red for the SZ state. The bottom plot in Figure 5 shows a bar graph where each bar, corresponding to one second, is colored depending on the answer of the CNN: a blue bar represents a pattern classified as NS, whereas a red bar characterizes a SZ pattern. By comparing the bars with the raw signals during the temporal evolution of the EEG recording a very good correspondence is found.

These results, showing a good performance of the CNN, are obtained from models that have been trained using a number of seizures. However, in case of a new patient, instead of collecting a sufficient number of seizures to customize a new patient-specific model from scratch (which sometimes is difficult) transfer-learning capabilities can be used. Starting from a model already trained for other patients, it may be retrained with new seizures of the new patient, obtaining a cross-patient seizure detection system with transfer learning among the patients.
3.2. Transfer Learning for Cross-Patient Seizure Detection. Transfer learning is a technique consisting of pretraining a model on one set of data and then fine-tuning it on another. In this case, the deep cross-patient transfer learning framework is applied to classify EEG data of a patient using the CNN trained with the EEG of another subject. In particular, starting from a patient-specific CNN model, its weights are used as the initial values for a second phase of training using seizures of another patient. This could provide many advantages when few seizures are available.

As an example, the patient-specific CNN model of patient \#9 (CNN9) has been assumed as the starting model. This choice has been done because on one hand patient \#9 has the highest number of seizures and on the other its model achieves the lowest performance in terms of Gmean.

Table 6 shows the results of the cross-patient seizure detection procedure applied to detect the seizures of patient \#5 starting from the network pretrained on patient \#9; Figure 6 reports the trends of Sensitivity and Specificity during the temporal occurrence of the five seizures of patient \#5, for the training set (blue line) and for the validation set (red line). The green squares in the same figure represent the performance obtained on the test seizures.

Starting from the CNN9 model, the first of the five seizures of patient $\# 5$ are used as a test case. As expected, the performance is quite low because the model has not been yet customized to the new patient. In particular, the CNN9 


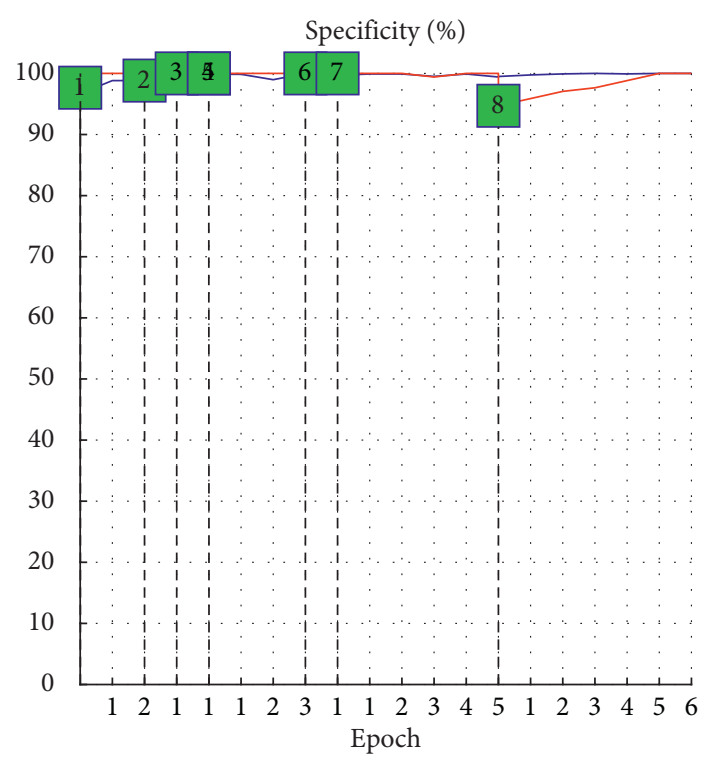

(a)

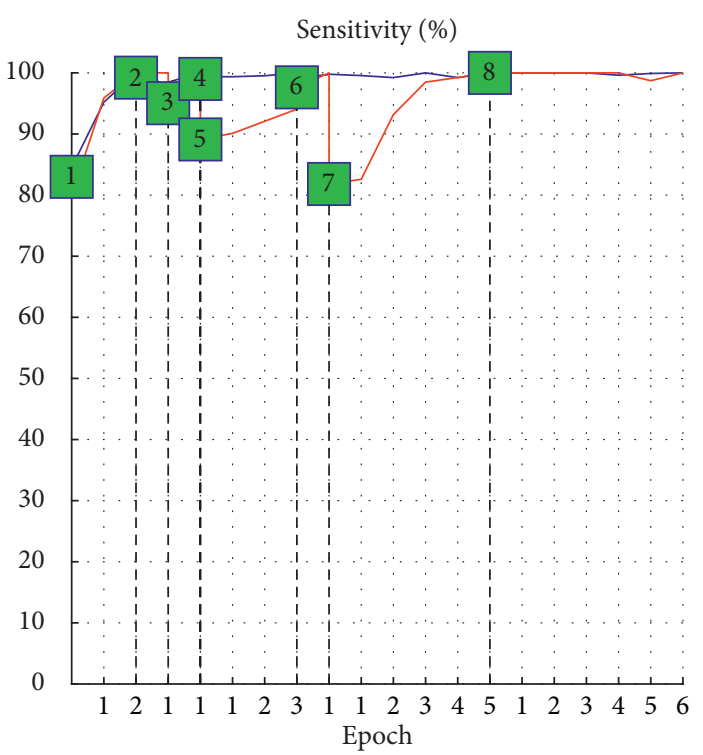

(b)

FigURE 7: Results of the cross-patient seizure detection procedure in terms of Specificity (a) and Sensitivity (b), starting from the seizure detection model of patient \#9: trend of Specificity and Sensitivity during the temporal occurrence of the 8 seizures of the patient \#6. Blue line: trend on the training set; red line: trend on the validation set; green squares: results on the test set.

TABle 7: Steps of the cross-patient seizure detection process for patient \#5.

\begin{tabular}{|c|c|c|c|c|c|}
\hline & Training SZ & Validation SZ & Test SZ & \# Epochs & CPU time (s) \\
\hline Step 0 & - & - & 1 & 0 & 0 \\
\hline Step 1 & 1 & 1 & 2 & 1 & 14 \\
\hline Step 2 & 1 & 2 & 3 & 0 & 454 \\
\hline Step 3 & 1,2 & 3 & 4 & 0 & 960 \\
\hline Step 4 & $1,2,3$ & 4 & 5 & 2 & 1528 \\
\hline
\end{tabular}

TABle 8: Test Performance (\%) of the cross-patient seizure detection system on patient \#6.

\begin{tabular}{lcccccccc}
\hline & SZ \#1 & SZ \#2 & SZ \#3 & SZ \#4 & SZ \#5 & SZ \#6 & SZ \#7 & SZ \#8 \\
\hline Accuracy & 88.53 & 99.26 & 97.75 & 99.63 & 95.94 & 98.82 & 92.07 & 96.37 \\
Specificity & 97.65 & 98.82 & 100 & 100 & 100 & 100 & 100 \\
Sensitivity & 83.22 & 100 & 95.04 & 99.01 & 89.11 & 97.63 & 81.82 \\
Gmean & 90.14 & 99.41 & 97.49 & 99.50 & 94.40 & 98.81 & 90.71 \\
\hline
\end{tabular}

model performs a low specificity (about 28\%), whereas the value of sensitivity is very high (100\%). This suggests refining the starting model by using patient-specific information.

Hence, in the next step, two-thirds of the EEG segments of the first seizure have been used as the training set and the other one-third as validation set, whereas the performance of the customized model has been tested on the second seizure, showing an improvement of specificity that increases to $85.92 \%$, while sensitivity is not degraded. In the subsequent steps, the last seizure has been used to test the model customized on the preceding seizures. As it can be noted, during the testing of the subsequent seizures, Specificity and Sensitivity values fluctuate keeping always above $80 \%$ and providing an ever high Gmean value up to the value of about $99 \%$ in the last tested seizure.

For each step of the procedure, Table 7 reports a summary of the cross-patient seizure detection process: the seizures used for training, validation, and test, the number of epochs performed during the training, and the total CPU time in seconds. If the number of epochs is zero, it means that, during the training, no network configuration has been found that improves the prediction accuracy on the validation step. Note that the process took less than 30 minutes to fine-tuning the model with each new data pattern.

Table 8 and Figure 7 reports the same results of Table 6 and Figure 6 but referring to patient \#6, starting again from the CNN9 model.

In this case, the cross-patient seizure detection system performs very well since the first seizure, especially concerning the specificity, which always takes values above about $95 \%$.

This fluctuating trend, especially concerning the sensitivity, demonstrates the diversity of seizures not only among patients but also within the same patient, which challenges all classifiers, whatever deep they may be. 
TABLE 9: Steps of the cross-patient seizure detection process for patient \#6.

\begin{tabular}{|c|c|c|c|c|c|}
\hline & Training SZ & Validation SZ & Test SZ & \# Epochs & CPU time (s) \\
\hline Step 0 & - & - & 1 & 0 & 0 \\
\hline Step 1 & 1 & 1 & 2 & 2 & 17 \\
\hline Step 2 & 1 & 2 & 3 & 1 & 12 \\
\hline Step 3 & 1,2 & 3 & 4 & 1 & 622 \\
\hline Step 4 & $1,2,3$ & 4 & 5 & 0 & 817 \\
\hline Step 5 & $1,2,3,4$ & 5 & 6 & 3 & 1206 \\
\hline Step 6 & $1,2,3,4,5$ & 6 & 7 & 1 & 32 \\
\hline Step 7 & $1,2,3,4,5,6$ & 7 & 8 & 5 & 415 \\
\hline
\end{tabular}

In Table 9, the transfer-learning process from CNN9 to patient \#6 is shown, with the same information reported in Table 7. Also, in this case, retraining the CNN9 with a new seizure takes no more than 20 minutes.

\section{Conclusions}

A CNN architecture has been deployed, by adapting the ResNet structure to the problem of seizure detection from EEG traces in nocturnal frontal lobe epilepsy (NFLE). The adjustment in the architecture consisted mainly in using $1 \mathrm{D}$ filters in the convolutional layers, to filter separately the information coming from different electrodes, and including dropout layers to reduce overfitting on the training set and improve generalization. An advantage of the deep-learning approach is that the complex and time-consuming feature engineering step is not required. Moreover, the developed patient-specific detection systems have demonstrated to be superior to those in the literature towards all the performance indexes, with accuracy above $94 \%$ for all the considered patients. In our knowledge, this is the first application of deep learning to NFLE seizure detection. Moreover, using the potentiality of the transfer learning, the lack of an extensive EEG database has been overcome and the possibility to develop a cross-patient seizure detection system has been demonstrated. Indeed, a patient-dependent system can be fine-tuned to a new patient with few data and limited computational effort.

\section{Data Availability}

Data were recorded on behalf of the EPILEPSIAE project.

\section{Consent}

Prior to admission, all patients were informed about the study and each one gave informed consent.

\section{Conflicts of Interest}

The authors confirm that there are no known conflicts of interest associated with this publication and there has been no significant financial support for this work that could have influenced its outcome.

\section{Acknowledgments}

The authors would like to acknowledge EU FP 7 Project EPILEPSIAE Grant 211713. This work was supported by the
Open Access Publishing Fund of the University of Cagliari, with the funding of the Regione Autonoma della Sardegna-L.R. n. 7/2007.

\section{References}

[1] F. Provini, G. Plazzi, P. Tinuper, S. Vandi, E. Lugaresi, and P. Montagna, "Nocturnal frontal lobe epilepsy: a clinical and polygraphic overview of 100 consecutive cases," Brain, vol. 122, no. 6, pp. 1017-1031, 1999.

[2] P. Ryvlin, S. Rheims, and G. Risse, "Nocturnal frontal lobe epilepsy," Epilepsia, vol. 47, no. 2, pp. 83-86, 2006.

[3] F. Rosenow, K. M. Kleim, and H. M. Hamer, "Non-invasive EEG evaluation in epilepsy diagnosis," Expert Rev Neurother. Expert Review of Neurotherapeutics, vol. 15, no. 4, 2015.

[4] U. R. Acharya, S. Vinitha Sree, G. Swapna, R. J. Martis, and J. S. Suri, "Automated EEG analysis of epilepsy: a review," Knowledge-Based Systems, vol. 45, pp. 147-165, 2013.

[5] O. Faust, U. R. Acharya, H. Adeli, and A. Adeli, "Waveletbased EEG processing for computer-aided seizure detection and epilepsy diagnosis," Seizure, vol. 26, pp. 56-64, 2015.

[6] D. Chen, S. Wan, J. Xiang, and F. S. Bao, "A high-performance seizure detection algorithm based on Discrete Wavelet Transform (DWT) and EEG," Plos One, vol. 12, no. 3, Article ID e0173138, 2017.

[7] M. Bandarabadi, C. A. Teixeira, F. Sales, and A. Dourado, "Wepilet, optimal orthogonal wavelets for epileptic seizure prediction with one single surface channel," IEEE Engineering in Medicine and Biology Society, vol. 2011, pp. 7059-7062, 2011.

[8] M. Zabihi, S. Kiranyaz, A. B. Rad, A. K. Katsaggelos, M. Gabbouj, and T. Ince, "Analysis of high-dimensional phase space via poincaré section for patient-specific seizure detection," IEEE Transactions on Neural Systems and Rehabilitation Engineering, vol. 24, no. 3, pp. 386-398, 2016.

[9] U. R. Acharya, H. Fujita, V. K. Sudarshan, S. Bhat, and J. E. W. Koh, "Application of entropies for automated diagnosis of epilepsy using EEG signals: a review," KnowledgeBased Systems, vol. 88, pp. 85-96, 2015.

[10] L. Wang, W. Xue, Y. Li et al., "Automatic epileptic seizure detection in EEG signals using multi-domain feature extraction and nonlinear analysis," Entropy, vol. 19, no. 6, p. 222, 2017.

[11] Y. Park, L. Luo, K. K. Parhi, and T. Netoff, "Seizure prediction with spectral power of EEG using cost-sensitive support vector machines," Epilepsia, vol. 52, no. 10, pp. 1761-1770, 2011.

[12] A. Kavitha and V. Krishnaveni, "A novel automatic stepwise signal processing based computer aided diagnosis system for epilepsy-seizure detection and classification for EEG," Biomedical Research, vol. 10, 2016. 
[13] P. Swami, T. K. Gandhi, B. K. Panigrahi, M. Tripathi, and S. Anand, "A novel robust diagnostic model to detect seizures in electroencephalography," Expert Systems with Applications, vol. 56, pp. 116-130, 2016.

[14] K. Cuppens, L. Lagae, B. Ceulemans, S. Van Huffel, and B. Vanrumste, "Detection of nocturnal frontal lobe seizures in pediatric patients by means of accelerometers: a first study," in Proceedings of the 31st Annual International Conference of the IEEE EMBS, Minneapolis, MA, USA, September 2009.

[15] T. M. E. Nijsen, J. B. A. M. Arends, P. A. M. Griep, and P. J. M. Cluitmans, "The potential value of three-dimensional accelerometry for detection of motor seizures in severe epilepsy," Epilepsy \& Behavior, vol. 7, no. 1, pp. 74-84, 2005.

[16] T. M. E. Nijsen, R. M. Aarts, P. J. M. Cluitmans, and P. A. M. Griep, "Time-frequency analysis of accelerometry data for detection of myoclonic seizures," IEEE Transactions on Information Technology in Biomedicine, vol. 14, no. 5, pp. 1197-1203, 2010.

[17] B. Pisano, B. Cannas, G. Milioli et al., "Autosomal dominant nocturnal frontal lobe epilepsy seizure characterization through wavelet transform of EEG records and self organizing maps," in Proceedings of the 2016 IEEE 26th International Workshop on Machine Learning for Signal Processing (MLSP), pp. 1-6, Vietri sul Mare, Italy, September 2016.

[18] H. H. Jasper, "Report of the committee on methods of clinical examination in electroencephalography," Electroencephalography and Clinical Neurophysiology, vol. 10, no. 2, pp. 370375, 1958.

[19] M. Bonnet, "EEG arousals: scoring rules and examples," Sleep, vol. 15, no. 2, pp. 173-184, 1992.

[20] B. Pisano, C. A. Teixeira, A. Dourado, and A. Fanni, "Application of Self Organizing Map to Identify Nocturnal Epileptic Seizures," Neural Computing and Applications, vol. 10, 2019.

[21] G. Litjens, T. Kooi, B. E. Bejnordi et al., "A survey on deep learning in medical image analysis," Medical Image Analysis, vol. 42, pp. 60-88, 2017.

[22] S. Pouyanfar, "A survey on deep learning: algorithms, techniques, and applications," ACM Computing Surveys, vol. 51, no. 5, pp. 92-36, 2018.

[23] M. Z. Alom, "A State-of-the-Art Survey on Deep Learning Theory and Architectures," Electronics, vol. 8, 2019.

[24] U. R. Acharya, S. L. Oh, Y. Hagiwara, J. H. Tan, and H. Adeli, "Deep convolutional neural network for the automated detection and diagnosis of seizure using EEG signals," Computers in Biology and Medicine, vol. 100, pp. 270-278, 2018.

[25] University of Bonn, Bonn Epilepsiae Data Base, University of Bonn, Bonn, Germany, 2019.

[26] I. Ullah, M. Hussain, E.-U.-H. Qazi, and H. Aboalsamh, “An automated system for epilepsy detection using EEG brain signals based on deep learning approach," Expert Systems With Applications, vol. 107, pp. 61-71, 2018.

[27] A. Emami, N. Kunii, T. Matsuo, T. Shinozaki, K. Kawai, and H. Takahashi, "Seizure detection by convolutional neural network-based analysis of scalp electroencephalography plot images," NeuroImage: Clinical, vol. 22, Article ID 101684, 2019.

[28] K. Simonyan and A. Zisserman, "Very deep convolutional networks for large-scale image recognition," in Proceedings of the ICLR 2015, International Conference on Learning Representations, pp. 1-14, San Diego, CA, USA, May 2015.

[29] X. Wei, L. Zhou, Z.. : Chen, L. Zhang, and Y. Zhou, "Automatic seizure detection using three dimensional CNN based on multi-channel EEG," BMC Medical Informatics and Decision Making, vol. 18, no. 5, p. 111, 2018.

[30] S. J. Pan and Q. Yang, "A survey on transfer learning," IEEE Transactions on Knowledge and Data Engineering, vol. 22, no. 10, pp. 1345-1359, 2010.

[31] I. Goodfellow, Y. Bengio, and A. Courville, Deep Learning (15.2 Transfer Learning and Domain Adaptation), MIT Press, Cambridge, MA, USA, 2016.

[32] J. Klatt, H. Feldwisch-Drentrup, M. Ihle et al., "The EPILEPSIAE database: an extensive electroencephalography database of epilepsy patients," Epilepsia, vol. 53, no. 9, pp. 1669-1676, 2012.

[33] K. He, "American Electroencephalographic Society guidelines for standard electrode position nomenclature," Journal of Clinical Neurophysiology, vol. 8, no. 2, pp. 200-202, 1991.

[34] K. He, X. Zhang, S. Ren, and J. Sun, "Deep residual learning for image recognition," in Proceedings of the IEEE Conference on Computer Vision and Pattern Recognition (CVPR), pp. 770-778, Las Vegas, NV, USA, 2016.

[35] N. Qian, "On the momentum term in gradient descent learning algorithms," Neural Networks, vol. 12, no. 1, pp. 145-151, 1999.

[36] T. N. Alotaiby, S. A. Alshebeili, T. Alshawi, I. Ahmad, and F. E. A. El-Samie, "EEG seizure detection and prediction algorithms: a survey," EURASIP Journal on Advances in Signal Processing, vol. 2014, no. 1, p. 183, 2014. 